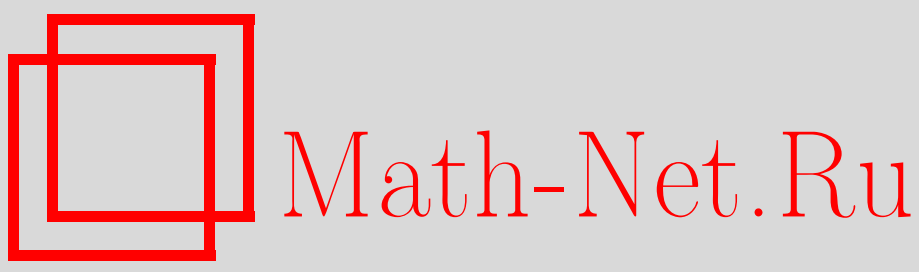

Д. И. Пионтковский, О рядах Гильберта и гомологиях PI-алгебр, Матем. сб., 1998, том 189, номер 11, 103-120

DOI: https://doi.org/10.4213/sm373

Использование Общероссийского математического портала Math-Net.Ru подразумевает, что вы прочитали и согласны с пользовательским соглашением

http: //www.mathnet.ru/rus/agreement

Параметры загрузки:

IP : 54.172 .240 .79

26 апреля 2023 г., 07:40:37 
УДК $512.66,512.552,512.554$

\section{Д.И. Пионтковский}

\section{О рядах Гильберта и гомологиях РІ-алгебр}

Основная цель этой статьи - обобщение хорошо известного в коммутативной алгебре критерия полных пересечений на случай произвольного многообразия PI-(супер)алгебр. В качестве следствия получены формулы для вычисления рядов Гильберта четной и нечетной частей относительно свободных супералгебр и свободных специальных йордановых супералгебр.

Библиографияя: 16 названий.

\section{§1. Введение}

В классической коммутативной алгебре важную роль играет теорема, называемая критерием полных пересечений. Всякой последовательности элементов локального или градуированного коммутативного кольца $A$ ставится в соответствие свободная дифференциально градуированная супералгебра над $A$ (комплекс Козюля). Критерий полных пересечений гласит, что последовательность регулярна тогда и только тогда, когда комплекс Козюля ацикличен, т.е. составляет минимальную резольвенту для факторкольца $A$ по идеалу, порожденному этой последовательностью элементов [1]. Последовательность регулярна, если каждый следующий ее элемент - не делитель нуля по модулю предыдущих; для градуированной алгебры $A$ это условие равносильно тому, что среди всех факторов по идеалам, порожденным однородными элементами с одной и той же последовательностью степеней, ряд Гильберта факторалгебры по регулярной последовательности минимален (т.е. размерность каждой градуированной компоненты наименьшая из возможных).

Как алгебра комплекс Козюля изоморфен внешней алгебре над $A$. Несложно обобщить такую конструкцию на случай коммутативной супералгебры $A$ (это было проделано Т. Юзефьяком [2]). Соответствующий аналог комплекса Козюля по-прежнему является свободной дифференциально градуированной алгеброй над $A$, однако теперь ее порождающие могут быть как нечетными, так и четными элементами - в зависимости от четности соответствующих элементов последовательности. Определение регулярной последовательности несколько изменяется, однако критерий полных пересечений остается верным: регулярность последовательности равносильна ацикличности комплекса Козюля и минимальности ряда Гильберта.

Работа выполнена при частичной поддержке Российского фонда фундаментальных исследований (грант № 96-01-00149). 
Аналог комплекса Козюля для произвольной ассоциативной алгебры над полем был построен в [3] и носит название комплекса Шафаревича. Соответствующий аналог понятия регулярной последовательности - это введенное Д. Аником понятие сильно свободного [4], или инертного [5], множества: множество однородных элементов ассоциативной алгебры $A$ называется сильно свободным (инертнылм), если, во-первых, оно составляет систему свободных образующих для порожденной им подалгебры и, во-вторых, $A$ как градуированное пространство изоморфна свободному произведению этой подалгебры и соответствующей факторалгебры. Это свойство равносильно ацикличности комплекса Шафаревича, а соответствуюшее неравенство для рядов Гильберта тесно связано с теоремой Голода-Шафаревича [3]. Отметим, что, как показал Е. С. Голод [6], комплекс Шафаревича обладает многими замечательными гомологическими свойствами, в том числе не имеющими аналогов в коммутативном случае.

Для случая градуированной супералгебры Ли аналогичные результаты были получены С. Гальпериньм и Ж.-М. Лемэром [7]. Аналогом регулярной последовательности служит инертное множество - подмножество градуированной алгебры Ли $L$, образ которого в универсальной обертывающей является инертным множеством в смысле Аника. Аналог комплекса Козюля для множества $\rho$ градуированных элементов из $L$ - дифференциально градуированная алгебра Ли, полученная из $L$ добавлением новых свободных порождающих, четность которых противоположна четности соответствующих элементов множества $\rho$. Над полем характеристики нуль ацикличность такого комплекса равносильна тому, что множество $\rho$ инертно. При этом для любого множества $\rho$ ряд Гильберта алгебры $A$ покоэффициентно меньше или равен ряду Гильберта алгебры, равной свободному произведению (в категории супералгебр Ли) свободной супералгебры Ли, порожденной $\rho$, и соответствующей факторалгебры: равенство равносильно тому, что $\rho$ инертно. Краткий список таких результатов, обобщающих коммутативные конструкции на суперкоммутативный, ассоциативный и лиев случаи, объединены ниже в теореме 5. Как ассоциативный, так и лиев варианты инертных множеств оказались полезными в алгебраической топологии, а также в теории групп (см. [4], [5], [7]).

Рассмотренные случаи коммутативных, ассоциативных и лиевых алгебр объединены тем, что все эти классы являются многообразиями алгебр с полиномиальными тождествами. В этой связи возникает вопрос: можно ли построить аналог комплекса Козюля для произвольного многообразия алгебр? Такой комплекс был построен (для многообразия ассоциативных алгебр при условии, что $A$ относительно свободна) А. А. Бояркиным [8]. В то время еще не были введены восходящие к работе А.Р. Кемера [9] многообразия супералгебр, и алгебраическая структура на этом комплексе определяется таким образом, чтобы идеал соотношений получившейся алгебры ( "кососвободной”) был дифференциальным идеалом. Впрочем, как будет показано ниже, эта супералгебра является относительно свободной и в обычном смысле. Связь ацикличности такого комплекса с деформациями и расширениями РІ-алгебр исследовалась А. А. Бояркиным, в частности в [10].

Мы будем рассматривать многообразия (супер)алгебр (вообе говоря, неассо-

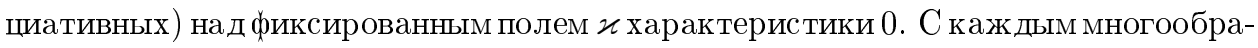
зием $W$ связана категория связно градуированных (т.е. конечно градуированных 
в положительных степенях) алгебр из $W .{ }^{1}$

В $\S 2$ ниже обсуждаются различные определения относительно свободной супералгебры многообразия. Оказывается, что описания ассоциативных относительно свободных супералгебр, которые появляются из работ А.Р. Кемера [9] и А.А. Бояркина [8], согласованы и легко обобщаются на неассоциативный случай. Это позволяет определить для всякого множества $\rho$ однородных элементов градуированной $W$-(супер)алгебры $A$ соответствуюший аналог комплекса Козюля

$$
K^{W}(A, \rho),
$$

который мы называем по аналогии с модельньм ассоциативным случаем комплексом ШІафаревича (§3). В том же $\S 3$ даны необходимые и достаточные условия конечности такого комплекса: важнейшее из них состоит в том, что для всякого конечного множества $\rho$ элементов произвольной градуированной алгебры $A \in W$ комплекс $K^{W}(A, \rho)$ конечен тогда и только тогда, когда в $W$ выполняется система тождеств Капелли некоторого порядка.

В $\S 4$ доказывается основное утверждение этой статьи - PI-аналог критерия полных пересечений (теорема 7). Соответствуюшее неравенство для рядов Гильберта доказывается в начале этого раздела, а условия равенства в нем (которые можно считать определением $W$-регулярной последовательности) даются леммой 4. Критерий полных пересечений для множества $\rho$, порождаюшего в $A$ идеал $I$, формулируется в терминах ацикличности комплекса Шафаревича, однако не для самой алгебры $A$, а для алгебры, ассоциированной с ней относительно $I$-адической фильтрации. Впрочем, как показано в конце этого параграфа, для многообразий ассоциативных и коммутативных (супер)алгебр ацикличность одного из этих комплексов легко получается из ацикличности другого при рассмотрении стандартных спектральных последовательностей.

$\mathrm{B} \S \S 5,6$ с помощью такой резольвенты вычисляются ряды Гильберта четной и нечетной части относительно свободных супералгебр (для случая, когда нечетных порождаюших не меншше чем четных), а также свободных специальных йордановых супералгебр. В частности, если многообразие таково, что ряды Гильберта относительно свободных супералгебр рациональны, то и ряды Гильберта таких четных и нечетных частей также рациональны.

Я хотел бы выразить благодарность за помощь в работе моему научному руководителю Е. С. Голоду.

\section{§2. Многообразия РІ-алгебр и супералгебр}

Мы будем рассматривать многообразия алгебр (вообше говоря, неассоциативных) над фиксированньм полем $\varkappa$ характеристики 0. Введем обозначения для самых распространенных многообразий:

Com - коммутативные алгебры,

Ass - ассоциативные алгебры,

\footnotetext{
${ }^{1} \mathrm{C}$ некоторьми многообразиями можно связать и категорию связно градуированных унитарных алгебр. При этом все наши результаты по существу не изменятся, лишь к алгебрам добавятся единицы, а к их рядам Гильберта - единичные свободные члены.
} 
Lie - алгебры Ли,

All - все алгебры.

Через $F^{W}(X)$ будем обозначать свободную алгебру многообразия $W$ с множеством порождающих $X$.

С каждым многообразием $W$ связано многообразие супералгебр, которые называются $W$-супералгебрами. В отличие от [11] здесь предполагается, что пересечение четной и нечетной частей в супералгебрах тривиально.

ОПреДЕЛЕНИЕ 1 [11]. Пусть $A=A_{0} \oplus A_{1}$, где $A_{i} A_{j} \in A_{i+j}, i, j \in \mathbb{Z}_{2},-$ супералгебра, $W$ - многообразие. Алгебра $A$ называется $W$-супералгеброй, если ее грассманова оболочка

$$
G(A)=G_{0} \otimes A_{0}+G_{1} \otimes A_{1}
$$

принадлежит $W$, где $G_{0}$ и $G_{1}$ - четная и нечетная части счетнопорожденной алгебры Грассмана $G$.

Нам потребуются явные конструкции свободных супералгебр многообразий, которые обобщают ассоциативные конструкции А. А. Бояркина и А.Р. Кемера.

Пусть $X=Y \sqcup Z$, где $Y$ и $Z$ - счетные множества, элементы которых мы будем называть соответственно четными и нечетными переменными. Рассмотрим $I_{W}-$ $T$-идеал в $F^{\mathrm{All}}(X)$, соответствуюший некоторому многообразию $W$.

Если

$$
\begin{aligned}
& f\left(y_{1}, \ldots, y_{m} ; z_{1}, \ldots, z_{n}\right) \\
& =\sum_{\substack{u=\left(u_{1}, \ldots, u_{n+1}\right), u_{j} \text { не зависят от } z_{1}, \ldots, z_{n}}} \sum_{\sigma \in S_{n}} \sum_{i} \alpha_{\sigma, u}^{i} u_{1} z_{\sigma(1)} u_{2} \cdots z_{\sigma(n)} u_{n+1}
\end{aligned}
$$

- полилинейный многочлен из $I_{W}$ (здесь $y_{1}, \ldots, y_{m} \in Y, z_{1}, \ldots, z_{n} \in Z$ и слагаемые в последней сумме могут различаться коэффициентами и расстановкой скобок), то через

$$
f^{*}\left(y_{1}, \ldots, y_{m} ; z_{1}, \ldots, z_{n}\right)
$$

обозначим (cp. [9]) полилинейный многочлен

$$
f^{*}=\sum_{u} \sum_{\sigma \in S_{n}} \sum_{i}(-1)^{\sigma} \alpha_{\sigma, u}^{i} u_{1} z_{\sigma(1)} u_{2} \cdots z_{\sigma(n)} u_{n+1}
$$

где расстановка скобок в слагаемых внутренней суммы та же, что и в соответствующих слагаемых в (1).

Если $\mathscr{P}$ - множество полилинейных многочленов от элементов $X$, то пусть $I_{W^{-}}:=I_{W}^{*}$ - идеал в $F^{\mathrm{All}}(X)$, порожденный элементами из $\left(I_{W} \cap \mathscr{P}\right)^{*}$, в которые в качестве аргументов подставлены всевозможные элементы соответствующих четностей. Тогда счетнопорожденная свободная $W$-супералгебра определяется как

$$
F^{W^{-}}(X)=F^{\mathrm{All}}(X) / I_{W^{-}}
$$


(А.А. Бояркин [8] назвал такие алгебры кососвободныцми; видимо, более естественно выражение относительно свободные супералгебры). Соответствие такого описания определению 1 следует из результатов А.Р. Кемера [9; §2]: его рассуждения легко переносятся на неассоциативный случай.

Множество всех гомоморфных образов (при гомоморфизмах, сохраняюших $\mathbb{Z}_{2}$-градуировку) такой алгебры мы будем называть многообразием $W$-супералгебр и обозначать через $W^{-}$, а гомоморфные образы при $\mathbb{Z}$-градуированных (и по четным, и по нечетным переменньм) гомоморфизмах нулевой степени назовем градуированными $W$-супералгебрами: они будут $\mathbb{Z}$-градуированными, если положить $\operatorname{deg} 1=\operatorname{deg} y_{i}=0, \operatorname{deg} z_{i}=1$. Если на некоторой алгебре $A$ имеется градуировка, преврашаюшая ее в градуированную $W$-супералгебру, то будем называть такую градуировку $W^{-}$-градуировкой, или просто суперградуировкой.

Заметим, что $\mathrm{All}^{-}=\mathrm{All}, \mathrm{Ass}^{-}=$Ass и всегда существует естественное вложение $W \subset W^{-}$, сопоставляющее каждой $W$-алгебре алгебру с тривиальной нечетной частью.

Для двух $W$-супералгебр $A$ и $B$ естественным образом определяется свободное произведение $A \stackrel{W^{-}}{*} B$ в $W^{-}$. А именно, пусть $A=F_{1} / I_{1}, B=F_{2} / I_{2}$, где $F_{1}, F_{2}$ - копии относительно свободной супералгебры $F^{W^{-}}(X)$ с порождающими $X_{1}=$ $Y_{1} \sqcup Z_{1}$ и $X_{2}=Y_{2} \sqcup Z_{2}$ соответственно. Положим тогда $Y=Y_{1} \sqcup Y_{2}, Z=Z_{1} \sqcup Z_{2}$, $X=Y \sqcup Z$ и

$$
A^{W_{*}^{-}} B=F^{W^{-}}(X) / J
$$

где $J$ - идеал в $F^{W^{-}}(X)$, порожденный множеством $I_{1} \cup I_{2}$. Отметим, что если $A, B \in W$, то это свободное произведение совпадает с обычньм свободным произведением в многообразии $W$.

Мы будем использовать и другое определение многочлена $f^{*}$, обобщающее "ассоциативное" определение А.А. Бояркина [8; определение 3.2.1].

Запишем

$$
f=f\left(x_{1}, \ldots, x_{N}\right)=\sum_{\sigma \in S_{N}} \sum_{i} \alpha_{\sigma}^{i} x_{\sigma(1)} \cdots x_{\sigma(N)} \in I_{W}
$$

где $x_{1}, \ldots, x_{N}$ - однородные элементы степеней $c_{1}, \ldots, c_{N}$ и слагаемые в последней сумме могут различаться коэффициентами и расстановкой скобок. Положим

$$
f^{*}=f^{*}\left(x_{1}, \ldots, x_{N}\right)=\sum_{\sigma \in S_{N}} \sum_{i} \alpha_{\sigma, c}^{i} x_{\sigma(1)} \cdots x_{\sigma(N)},
$$

где для каждого набора степеней $c=\left(c_{1}, \ldots, c_{N}\right), c_{i} \geqslant 0$, коэффициенты $\alpha_{\sigma, c}^{i}$ определяются рекуррентно:

$$
\begin{gathered}
\alpha_{\sigma, c}^{i}=\alpha_{\sigma}^{i}, \quad \text { если } c=(0, \ldots, 0), \\
\alpha_{\sigma, d}^{i}=\alpha_{\sigma, c}^{i} \cdot(-1)^{\sum_{j=1}^{\sigma^{-1}(l)-1} c_{\sigma(j)}+\sum_{j=1}^{l-1} c_{j}}, \quad d=c+(0, \ldots, 1, \ldots, 0) .
\end{gathered}
$$


ЛЕмма 1. Предложсенные определения многочлена $f^{*}$ эквивалентны.

ДокАЗАТЕЛЬСТво. Пусть $N=m+n$ и подстановка $\sigma \in S_{N}$ действует на множестве $\left\{x_{1}, \ldots, x_{N}\right\}$, элементам которого приписан вектор степеней $c=\left(c_{1}, \ldots, c_{N}\right)$; пусть среди этих элементов ровно $m$ имеют четные степени и ровно $n$ - нечетные. Суперзнаком перестановки $\sigma$ (обозначение: $\operatorname{ssg}_{c} \sigma$ ) будем называть знак соответствующей перестановки $\sigma^{\prime} \in S_{n}$, получающейся из $\sigma$ путем ограничения на множество переменных нечетной степени. Тогда формулу (2) можно переписать так:

$$
\begin{aligned}
& \text { если } f=\sum_{\sigma \in S_{N}} \sum_{i} \alpha_{\sigma}^{i} x_{\sigma(1)} \cdots x_{\sigma(N)} \in I_{W}, \\
& \text { то } \quad f^{*}=\sum_{\sigma \in S_{N}} \sum_{i} \alpha_{\sigma}^{i} \cdot(-1)^{\operatorname{ssg}_{c} \sigma} x_{\sigma(1)} \cdots x_{\sigma(N)} .
\end{aligned}
$$

Таким образом, достаточно доказать, что коэффициент $\alpha_{\sigma, c}^{i}$ в формулах (4) равен $\alpha_{\sigma}^{i} \cdot(-1)^{\operatorname{ssg}_{c} \sigma}$, т.е. при $d=c+(0, \ldots, 1, \ldots, 0)$

$$
\sum_{j=1}^{\sigma^{-1}(l)-1} c_{\sigma(j)}+\sum_{j=1}^{l-1} c_{j} \equiv \operatorname{ssg}_{c} \sigma+\operatorname{ssg}_{d} \sigma \quad(\bmod 2) .
$$

Это утверждение становится очевидным, если посмотреть, как меняется число инверсий нечетных элементов при изменении четности одного из них.

\section{§3. Комплекс Шафаревича в многообразиях супералгебр}

Пусть $W$ - многообразие, $A$ - связно градуированная $W$-супералгебра (т.е. суперградуировка на $A$ связна). Пусть $\rho=\left\{\rho_{i}\right\}_{i \in I}$ - набор однородных элементов из $A$ с вектором степеней $d=\left\{d_{i}\right\}_{i \in I}, d_{i} \geqslant 0$. Рассмотрим биградуированную $W$-супералгебру

$$
K^{W}(A, \rho)=A^{W^{-}} F^{W^{-}}(\gamma),
$$

где $\gamma=\left\{\gamma_{i}\right\}_{i \in I}, \operatorname{deg} \gamma_{i}=d_{i}+1$. Вторая $\mathbb{Z}$-градуировка на этой алгебре задается условиями

$$
\begin{gathered}
|a|=0, \quad a \in A, \\
\left|\gamma_{i}\right|=1
\end{gathered}
$$

(назовем ее гомологической градуировкой); в этом случае тотальная градуировка на $K^{W}(A, \rho)$ связна.

Пусть на $A$ задано однородное диф ференцирование $\partial_{A}$ степени - 1 , действуюшее по формуле Лейбница

$$
\partial_{A}(u v)=\partial_{A} u \cdot v+(-1)^{\operatorname{deg} u} u \cdot \partial_{A} v,
$$

где $u, v \in A$ - однородные элементы. 
ПреДЛОЖЕНИЕ 2. Существует однородное дифференцирование Ә на $K^{W}(A, \rho)$, имеющее степень -1 по обеим градуировкам, совпадающее с $\partial_{A}$ на $A$ и такое, что $\partial \gamma_{i}=\rho_{i}$.

Аналогичное утверждение было доказано А.А. Бояркиньм для относительно свободной алгебры $A$ [8; предложение 3.2.1].

ДокАЗАТЕЛЬСТво. Спервапостроим дифференцирование $\delta$, удовлетворяющее условиям предложения, в алгебре

$$
K^{\mathrm{All}}(A, \rho)=A_{*}^{\mathrm{All}^{-}} F^{\mathrm{All}^{-}}(\gamma) .
$$

Это легко сделать, определяя $\delta$ по формуле Лейбница с помощью индукции по гомологической градуировке. Заметим теперь, что

$$
K^{W}(A, \rho)=K^{\mathrm{All}}(A, \rho) / J
$$

где $J \triangleleft K^{\mathrm{All}}(A, \rho)$ - двусторонний идеал, порожденный всевозможными многочленами $f^{*}\left(x_{1}, x_{2}, \ldots\right)$, где $x_{1}, x_{2}, \ldots \in K^{\mathrm{All}}(A, \rho)$ - однородные относительно суперградуировки, $f$-полилинейное тождество $W$. Дифференцирование $\delta$ на $K^{\operatorname{All}}(A, \rho)$ индуцирует искомое дифференцирование $\partial$, если только $J$ - дифференциальный идеал, т.е. $\delta J \in J$.

Докажем это. Достаточно проверить, что образы порождающих попадают в идеал. Пусть $x_{1}, x_{2}, \ldots, x_{N} \in K^{\mathrm{All}}(A, \rho)$ - однородные относительно суперградуировки элементы с вектором степеней $c=\left(c_{1}, \ldots, c_{N}\right), f$-полилинейное тождество $W$ от $N$ переменных. По формулам (4) и (5) имеем:

$$
\begin{aligned}
\delta f^{*} & \left(x_{1}, \ldots, x_{N}\right) \\
& =\sum_{\sigma \in S_{N}} \sum_{i} \alpha_{\sigma, c}^{i} \delta\left(x_{\sigma(1)} \cdots x_{\sigma(N)}\right) \\
& =\sum_{\sigma \in S_{N}} \sum_{i} \alpha_{\sigma, c}^{i} \cdot(-1)^{\sum_{j=1}^{k-1} c_{\sigma(j)}} x_{\sigma(1)} \cdots\left(\delta x_{\sigma(k)}\right) \cdots x_{\sigma(N)} \\
& =\sum_{m=1}^{N} \sum_{k=1}^{N} \sum_{\sigma \in S_{N}, \sigma(k)=m} \sum_{i} \alpha_{\sigma, c}^{i} \cdot(-1)^{\sum_{j=1}^{k-1} c_{\sigma(j)}} x_{\sigma(1)} \cdots\left(\delta x_{m}\right) \cdots x_{\sigma(N)} .
\end{aligned}
$$

Положим $b=\left(c_{1}, \ldots, c_{m}-1, \ldots, c_{N}\right)$,

$$
y_{i}= \begin{cases}x_{i}, & i \neq m \\ \delta x_{m}, & i=m\end{cases}
$$

и вычислим отдельно тройную внутреннюю сумму. Если $c_{m}=0$, то она равна нулю (и, значит, лежит в $J$ ); в противном случае она равна

$$
\begin{aligned}
& \sum_{\sigma \in S_{N}, k=\sigma^{-1}(m)} \sum_{i} \alpha_{\sigma, c}^{i} \cdot(-1)^{\sum_{j=1}^{k-1} c_{\sigma(j)}} y_{\sigma(1)} \cdots y_{\sigma(k)} \cdots y_{\sigma(N)} \\
= & \sum_{\sigma \in S_{N}} \sum_{i} \alpha_{\sigma, b}^{i} \cdot(-1)^{\sum_{j=1}^{\sigma^{-1}(m)-1} c_{\sigma(j)}+\sum_{j=1}^{m-1} c_{j}+\sum_{j=1}^{\sigma^{-1}(m)-1} c_{\sigma(j)}} y_{\sigma(1)} \cdots y_{\sigma(N)} \\
= & \sum_{\sigma \in S_{N}} \sum_{i} \alpha_{\sigma, b}^{i} \cdot(-1)^{\sum_{j=1}^{m-1} c_{j}} y_{\sigma(1)} \cdots y_{\sigma(N)}=(-1)^{\sum_{j=1}^{m-1} c_{j}} f^{*}\left(y_{1}, \ldots, y_{N}\right) \in J
\end{aligned}
$$


(как и прежде, во всех выкладках в одинарной внутренней сумме слагаемые могут различаться расстановкой скобок). Предложение доказано.

Пусть $\partial_{A}=0$. Определяемое предложением 2 дифференцирование $\partial$ превращает $K^{W}(A, \rho)$ в дифференциально градуированную алгебру. Мы будем обозначать ее группы гомологий (относительно гомологической градуировки) через $H_{*}^{W}(A, \rho)$.

Алгебра $K^{\mathrm{Com}}(A, \rho)$ для $A \in \mathrm{Com}$ известна с 1950 года [12], а для $A \in \mathrm{Com}^{-}$ - с 1972 года [2] под названием комплекса Козюля. Алгебра $K^{\mathrm{Ass}}(A, \rho)$ носит название комплекса Шафаревича [3]: мы будем так же называть эту дифференциально градуированную алгебру и в случае произвольного многообразия $W$. Для $W \subset$ Ass алгебра $K^{W}\left(F^{W}(X), \rho\right)$ рассматривалась А. А. Бояркиньп под названием обобщенного комплекса Козюля.

В заключение этого параграфа, приведем условия конечности комплекса Шафаревича. Говорят, что в многообразии $W$ выполняется система тождеств Kaneлли некоторого порядка $n$, если для всякого полилинейного неассоциативного многочлена $f=f\left(x_{1}, \ldots, x_{n} ; y_{1}, y_{2}, \ldots\right)$, кососимметрического по переменным $x_{1}, \ldots, x_{n}$, в $W$ выполняется тождество $f \equiv 0$.

ПРЕДЛОЖЕНИЕ 3. Следующие условия на многообразие $W$ әквивалентнь:

(i) ВW выполняется система тождеств Капелли некоторого порядка $n+1$.

(ii) Для любой градуированной алгебрь $A$ из $W$ и любого конечного множества однородных әлементов $\rho \subset A$ комплекс Шафаревича $K^{W}(A, \rho)$ конечен.

(iii) Для любой градуированной супералгебры $A$ из $W^{-}$и любого конечного множества четных однородных элементов $\rho \subset A$ комплекс Шафаревича $K^{W}(A, \rho)$ конечен.

$B$ әтом случае длина комплекса $K^{W}(A, \rho)$ не превосходит $n \cdot \operatorname{Card} \rho$.

Если $W \subset \mathrm{Ass}$ - многообразие ассоциативных алгебр, то условия (i), (ii),

(iii) әквивалентны следующим условиям:

А. ВW выполняется стандартное тождество некоторого порядка.

В. Для некоторой градуированной алгебры $A$ из W найдется такое множество однородных әлементов $\rho \subset A$, что комплекс Шафаревича $K^{W}(A, \rho)$ конечен.

С. Для некоторой градуированной супералгебры $A$ из $W^{-}$найдется такое множество четных однородных әлементов $\rho \subset A$, что комплекс Шафаревича $K^{W}(A, \rho)$ конечен.

D. “Внешняя" алгебра ранга 1 (т.е. свободная W-супералгебра, порожденная единственной нечетной переменной) конечномерна.

ДоказАтельство. Импликация (iii) $\Rightarrow($ ii) очевидна. Докажем (i) $\Rightarrow$ (iii) и (ii) $\Rightarrow(\mathrm{i})$.

Условие (i) необходимо и достаточно проверять для $A=F^{W}(X)$, где $X$-счетное множество. Тогда если обозначать через $F_{m, l}$ свободную алгебру многообра- 
зия $W^{-}$с $m$ четными и $l$ нечетными порождающими, то $K^{W}(A, \rho) \simeq F_{\infty, s}$, где $s=\operatorname{Card} \rho$.

Таким образом, (i) равносильно тому, что в алгебре $F=F_{\infty, s}$ все слова, имеющие по нечетным порождающим степень, большую некоторого числа $N$, нулевые. В частности, в $W^{-}$выполняется супертождество

$$
\mathrm{c}\left(x ; y_{1}, y_{2}, \ldots\right)=\cdots x \cdots x \cdots x \cdots \equiv 0
$$

где нечетная переменная $x$ встречается ровно $N$ раз, а имеюшие произвольную четность переменные $y_{1}, y_{2}, \ldots-$ по одному разу. Линеаризуя, получаем, что в $W^{-}$выполняется супертождество

$$
\sum_{\sigma \in S_{N}} a\left(x_{\sigma(1)}, \ldots, x_{\sigma(N)} ; y_{1}, y_{2}, \ldots\right) \equiv 0
$$

где $a\left(x_{1}, \ldots, x_{N} ; y_{1}, y_{2}, \ldots\right)$ - такое полилинейное слово от $N$ переменных, что

$$
a\left(x, \ldots, x ; y_{1}, y_{2}, \ldots\right)=c\left(x ; y_{1}, y_{2}, \ldots\right)
$$

(здесь $x_{1}, \ldots, x_{N}$ нечетны, $y_{1}, y_{2}, \ldots-$ произвольной четности). Тем самым, в $W$ вьполняется тождество

$$
\sum_{\sigma \in S_{N}}(-1)^{\sigma} a\left(x_{\sigma(1)}, \ldots, x_{\sigma(N)} ; y_{1}, y_{2}, \ldots\right) \equiv 0
$$

для произвольного полилинейного неассоциативного слова $a$, т.е. вьполняется система тождеств Капелли порядка $N$. Тем самым доказана импликация (i) $\Rightarrow$ (iii).

Обратно, пусть в $W$ выполняется система тождеств Капелли порядка $n+1$. Тогда для всякого полилинейного неассоциативного слова

$$
a\left(x_{1}, \ldots, x_{n+1} ; y_{1}, y_{2}, \ldots\right)
$$

в $W$ выполняется тождество

$$
\sum_{\sigma \in S_{n+1}}(-1)^{\sigma} a\left(x_{\sigma(1)}, \ldots, x_{\sigma(n+1)} ; y_{1}, y_{2}, \ldots\right) \equiv 0
$$

а в $W^{-}-$супертождество

$$
\sum_{\sigma \in S_{n+1}} a\left(x_{\sigma(1)}, \ldots, x_{\sigma(n+1)} ; y_{1}, y_{2}, \ldots\right) \equiv 0
$$

при нечетных $x_{1}, \ldots, x_{n+1}$, откуда

$$
a\left(x, \ldots, x ; y_{1}, y_{2}, \ldots\right) \equiv 0
$$


для любого неассоциативного слова $a$, имеющего по нечетной переменной $x$ степень, большую $n$. Следовательно, степень любого ненулевого в $F$ слова по каждой из нечетных порождающих не превосходит $n$, и его степень по всем нечетньм переменньм не превосходит $n s$.

Импликация (i) $\Leftrightarrow$ А доказана А.Р. Кемером [13].

$\mathrm{A} \Leftrightarrow \mathrm{D}$. Алгебра $F_{0,1}$ конечномерна тогда и только тогда, когда в $W^{-}$при нечетном $x$ выполняется тождество $x^{N} \equiv 0$ для некоторого $N$, т.е. для нечетных $x_{1}, \ldots, x_{N}$ выполняется его линеаризация

$$
\sum_{\sigma \in S_{N}} x_{\sigma(1)} \cdots x_{\sigma(N)} \equiv 0 .
$$

Это супертождество эквивалентно обычному тождеству

$$
\sum_{\sigma \in S_{N}}(-1)^{\sigma} x_{\sigma(1)} \cdots x_{\sigma(N)} \equiv 0
$$

т.е. стандартному тождеству $\mathrm{St}_{N}$.

$\mathrm{D} \Rightarrow$ В. Пусть $A=\{0\}$. Тогда $K^{W}(A, \rho) \simeq F_{0,1}$.

$\mathrm{C} \Rightarrow \mathrm{D}$. Эта импликация следует из того, что $F_{0,1}^{W}$ - подалгебра во всякой алгебре

$K^{W}(A, \rho) \simeq A^{W^{-}} F_{0, \text { Card } \rho}$, если в множестве $\rho$ есть хотя бы один четный элемент.

Импликация $\mathrm{B} \Rightarrow \mathrm{C}$ очевидна: предложение доказано.

\section{§4. Ряды Гильберта и гомологии}

Пусть $A \in W^{-}-$связно градуированная супералгебра, $\rho=\left\{\rho_{1}, \rho_{2}, \ldots\right\} \subset A$ - множество однородных элементов с вектором степеней $d=\left\{d_{1}, d_{2}, \ldots\right\}, d_{i} \geqslant 1$, порождаюшее минимальным образом двусторонний идеал $J \triangleleft A$. Рассмотрим биградуированную алгебру

$$
\bar{A}: \bar{A}_{p, q}=\left(J^{p} / J^{p+1}\right)_{p+q}, \quad p \geqslant 0,
$$

где $J^{0}=A$, а при $p>0$ идеал $J^{p}$ порожден всевозможными произведениями $p$ элементов $J$. Пусть $Q=J /\left(A_{+} J+J A_{+}\right)$- векторное пространство, порожденное $\rho$, и $\pi: J / J^{2} \rightarrow Q$ - каноническая проекция. Если $\lambda: Q \rightarrow J / J^{2}-$ какой-нибудь гомоморфизм градуированных векторных пространств, обратный справа к $\pi$, то индуцированное им отображение

$$
\chi: A / J \stackrel{W^{-}}{*} F^{W^{-}}(\rho) \rightarrow A
$$

является сюръекцией биградуированных алгебр.

Если $A$ градуирована конечно, то, поскольку $\bar{A}$ с тотальной градуировкой изоморфна $A$ как гра дуированное векторное пространство, для рядов Гильберта справедливо покоэффициентное неравенство

$$
\left(A / J^{W^{-}} F^{W^{-}}(\rho)\right)(t) \geqslant A(t)
$$


Как следует из сказанного вьше, при этом справедлива

ЛЕмма 4. Следующие условия әквивалентны:

(i) неравенство (*) обращается в равенство;

(ii) для некоторого $\lambda$ отображение $\chi$ является изоморфизмом;

(iii) для любого $\lambda$ отображение $\chi$ является изоморфизмом.

Отметим, что, как показал Д. Аник [4; лемма 1.2, теорема 2.6], если $W=$ Ass и $A$ - свободная ассоциативная алгебра, то равенство в $(*)$ эквивалентно равенству в неравенстве Голода-Шафаревича [3].

В следуюшей теореме мы объединили несколько известных результатов.

Teорема 5. Пусть $W$ - одно из многообразий Com, Ass или Lie, и пусть $A \in W^{-}-$связная конечно градуированная супералгебра. Тогда следующие условия на мнохсество $\rho$ әквивалентны:

(i) $H_{1}^{W}(A, \rho)=0$;

(ii) $H_{0}^{W}(A, \rho)=A / J$ u $H_{i}^{W}(A, \rho)=0$ npu $i>0$;

(iii) неравенство (*) обращается в равенство:

$$
\left(A / J^{W^{-}} F^{W^{-}}(\rho)\right)(t)=A(t)
$$

Для $W=\operatorname{Com}$ и $A \in$ Com эта теорема эквивалентна классическому критерию полных пересечений (см. [1; §9, теорема 1], [14; следствие 3.2]): те множества $\rho$, для которых выполняются условия теоремы, называются регулярными. Для $W=\mathrm{Com}$ и $A \in \mathrm{Com}^{-}$аналог критерия полных пересечений был доказан Т. Юзефьяком [2]: он назвал такие множества градуированнымми регулярными или нормальными. При $W=$ Ass теорема 5 была доказана Д. Аником [4]: соответствующие множества $\rho$ названы им сильно свободными или инертнымми [5]. С. Гальперин и Ж.-М. Лемэр [7] доказали теорему 5 для супералгебр Ли: оказалось, что такие множества (авторы также называют их инертнымми) - в точности те, образы которых в универсальной обертывающей являются инертными множествами в смысле Аника.

Как показьвает следующая лемма, в произвольном многообразии $W$ аналог теоремы 5 справедлив для некоторых алгебр, для которых условие (iіi) выполняется автоматически.

ЛЕмма 6. Пусть $W$ - произвольное многообразие, $A \in W^{-}-$связная конечно градуированная супералгебра и, в прежсних обозначениях,

$$
G:=A / J^{W^{-}} F^{W^{-}}(\rho)
$$

Тогда $H_{0}^{W}(G, \rho)=A / J$ и $H_{i}^{W}(G, \rho)=0$ nри $i>0$. 
ДоказАтельство. Пусть $E=K^{W}(G, \rho)=A / J_{*}^{W^{-}} F^{W^{-}}(\rho) \stackrel{W^{-}}{*} F^{W^{-}}(\gamma)$, $\partial$ - дифференцирование этого комплекса Шафаревича, имеющее степень -1 по обеим градуировкам. Введем на $E$ новое дифференцирование $\delta$, имеющее степень -1 относительно суперградуировки и степень +1 относительно гомологической градуировки. Именно, положим

$$
\begin{aligned}
\delta(A / J) & =0 \\
\delta\left(\gamma_{i}\right) & =0, \\
\delta\left(\rho_{i}\right) & =\gamma_{i}
\end{aligned}
$$

и продолжим $\delta$ на всю алгебру $E$. Это можно сделать с помощью предложения 2, применив его к алгебре $G^{\prime}=A / J^{W^{-}} F^{W^{-}}(\gamma)$ и рассмотрев $E$ как комплекс Шафаревича $K^{W}\left(G^{\prime}, \gamma\right)$.

Зададим на $E$ еше одну градуировку: $\|a\|=0$ при $a \in A / J$ и $\left\|\gamma_{i}\right\|=\left\|\rho_{i}\right\|=1$. Относительно такой градуировки оба дифференцирования однородны нулевой степени. Как легко убедиться по индукции, для однородных относительно всех градуировок элементов $x \in E$

$$
(\delta \partial+\partial \delta) x=\|x\| \cdot x
$$

Определим теперь на комплексе $K_{+}^{W}(G, \rho)$ линейное отображение $g$, задав его на однородных относительно градуировки $\|\cdot\|$ элементах $x$ как

$$
g(x)=\frac{1}{\|x\|} \delta(x) .
$$

На подкомплексе $K_{+}^{W}(G, \rho)$ имеем:

$$
g \partial+\partial g=\mathrm{id}
$$

и, поскольку дифференциал гомотопен тождественному отображению, $H_{i}^{W}(G, \rho)$ $=0$ при $i>0$. Утверждение об $H_{0}$ тривиально: лемма доказана.

Для произвольной связно градуированной супералгебры $A \in W^{-}$критерий равенства в $(*)$ можно сформулировать в терминах комплекса Шафаревича для алгебры $\bar{A}$, которая является $W^{-}$-супералгеброй относительно тотальной градуировки.

ТЕОРема 7. Пусть $W$ - произвольное многообразие и $A \in W^{-}$- связная конечно градуированная супералгебра. Тогда следующие условия на множество $\rho$ әквивалентны:

(i) $H_{1}^{W}(\bar{A}, \rho)=0$;

(ii) $H_{0}^{W}(\bar{A}, \rho)=A / J$ u $H_{i}^{W}(\bar{A}, \rho)=0$ nрu $i>0$;

(iii) неравенство $(*)$ обращается в равенство:

$$
\left(A / J_{*}^{W^{-}} F^{W^{-}}(\rho)\right)(t)=A(t)
$$

Мы будем называть множества $\rho$, для которых выполняются условия этой теоремы, $W$-регулярными. 
ДокАЗАТЕЛЬСТво. Импликация (ii) $\Rightarrow$ (i) тривиальна, а импликация (iii) $\Rightarrow$ (ii) следует из лемм 4 и 6 . Докажем (i) $\Rightarrow$ (iii).

Пусть $G=A / J^{W^{-}} F^{W^{-}}(\rho), \gamma=\left\{\gamma_{1}, \gamma_{2}, \ldots: \operatorname{deg} \gamma_{i}=\operatorname{deg} \rho_{i}+1\right\}, F=G^{W^{-}}$ $F^{W^{-}}(\gamma), B=\bar{A}^{W^{-}} F^{W^{-}}(\gamma)$. Введем на $F$ и на $B$ гомологическую градуировку: $|G|=0,|\bar{A}|=0,\left|\gamma_{i}\right|=1$. Относительно нее сюръекция биградуированных алгебр

$$
h=\chi^{W^{-}} \text {id }: F \rightarrow B
$$

будет однородной нулевой степени.

Введем на $F$ дифференцирование $d: d(G)=0, d\left(\gamma_{i}\right)=\rho_{i}$ (это возможно сделать, поскольку алгебра $F$ изоморфна комплексу Шафаревича $\left.K^{W}(G, \rho)\right)$, а дифференцирование комплекса Шафаревича на $B$ обозначим через $\partial$. Эти дифференцирования однородны степени -1 по обеим градуировкам. Докажем, что $h$ является морфизмом комплексов, т.е. $h \circ d=\partial \circ h$.

Пусть $x \in F$ - элемент, однородный по обеим градуировкам. Если $x$ представим в виде линейной комбинации элементов из $A / J, \rho$ и $\gamma$ (в частности, если $\operatorname{deg} x \leqslant 1$ ), то, очевидно, $(h d) x=(\partial h) x$. В противном случае в силу линейности можно считать, что

$$
x=\sum_{i} \alpha_{i} a_{i} b_{i}, \quad \alpha_{i} \in \varkappa,
$$

где $\operatorname{deg} a_{i}<\operatorname{deg} x$ и $\operatorname{deg} b_{i}<\operatorname{deg} x$ для каждого $i$. Тогда по индукции можно считать, что

$$
(h d) a_{i}=(\partial h) a_{i}, \quad(h d) b_{i}=(\partial h) b_{i},
$$

и потому

$$
(h d) x=\sum_{i}\left((h d) a_{i} \cdot h b_{i}+(-1)^{\operatorname{deg} a_{i}} h a_{i} \cdot(h d) b_{i}\right)=(\partial h) x .
$$

Таким образом, $h: F \rightarrow B$ - морфизм нулевой степени биградуированных дифференциальных алгебр. Здесь $B=K^{W}(\bar{A}, \rho), F=A / J^{W^{-}} K^{W}\left(F^{W^{-}}(\rho), \rho\right)$ и согласно лемме 4

$$
H_{i}(F)= \begin{cases}0, & i>0 \\ A / J, & i=0\end{cases}
$$

Для нулевых относительно гомологической градуировки компонент имеем $F_{0}=G$, $B_{0}=\bar{A}, h_{0}=\chi$, и в следуюшей коммутативной диаграмме строки точны:

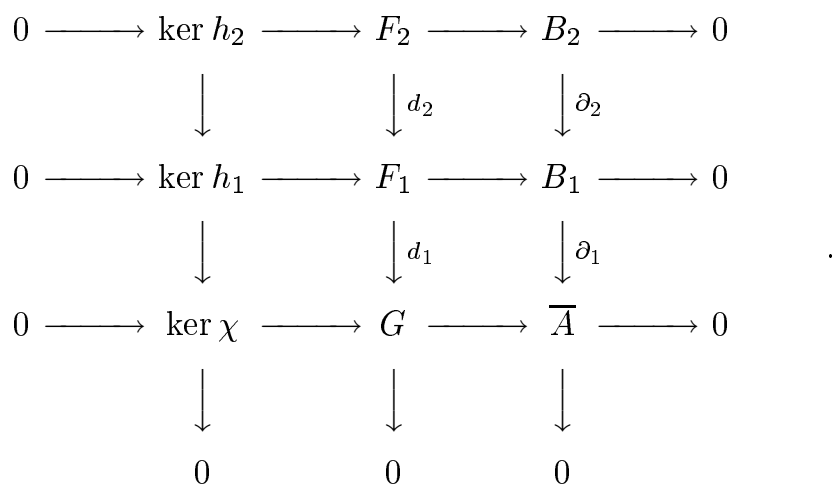


Поскольку $H_{1}(F)=0$, получаем

$$
0 \longrightarrow H_{1}(B) \stackrel{\partial_{*}}{\longrightarrow} \operatorname{ker} \chi / d\left(\operatorname{ker} h_{1}\right) \longrightarrow A / J \stackrel{\chi_{*}}{\longrightarrow} A / J \longrightarrow 0,
$$

где $\chi_{*}=\pi \lambda=\mathrm{id}$, откуда $0=H_{1}(B) \simeq \operatorname{ker} \chi / d\left(\operatorname{ker} h_{1}\right)$.

Пусть $\Delta=\operatorname{ker} \chi \in G$. Если $\Delta=0$, то $\chi-$ изоморфизм, и в силу леммы 4 вьполняется (iii). Предположим, что $\Delta \neq 0$.

Всякий элемент в ker $h_{1}$ представим в виде

$$
\sum_{j} \cdots \delta_{j} \cdots \gamma_{i_{j}} \cdots+\sum_{k} \cdots \gamma_{i_{k}} \cdots \delta_{k} \cdots
$$

где $\delta_{k} \in \Delta$, многоточия, возможно, заменяют произведения элементов из $G$ и слагаемые могут различаться множителями и расстановкой скобок. Тогда всякий элемент из $d\left(\operatorname{ker} h_{1}\right)$ представим в виде

$$
\sum_{j} \cdots \delta_{j} \cdots \rho_{i_{j}} \cdots+\sum_{k} \cdots \rho_{i_{k}} \cdots \delta_{k} \cdots
$$

Следовательно, если $l$ - наименьшая из степеней ненулевых элементов $\Delta$, $m=\min _{i} \operatorname{deg} \rho_{i}$, то степень любого ненулевого элемента $d\left(\operatorname{ker} h_{1}\right)$ не меньше $l+m>l$, откуда $d\left(\operatorname{ker} h_{1}\right) \neq \Delta$ и, в противоречие с (iii), $H_{1}(B) \neq 0$.

Замечание. Как видно из теорем 5 и 7 , для многообразий Com, Ass и Lie комплексы $K=K^{W}(A, \rho)$ и $\bar{K}=K^{W}(\bar{A}, \rho)$ ацикличны в положительных степенях одновременно. Для многообразий $W=\mathrm{Com}$ и $W=\mathrm{Ass}$, когда свободное произведение в $W$ - обычное свободное или тензорное произведение, в том, что из ацикличности комплекса $\bar{K}$ следует ацикличность $K$, можно убедиться и непосредственно.

Действительно, введем на комплексах $K$ и $\bar{K}$ убьваюшую фильтрацию по степеням идеала $N \triangleleft K$ (соответственно $\bar{N} \triangleleft \bar{K})$, порожденного подмножеством $\rho$ в $K$ (соответственно в $\bar{K})$. При этом индуцированная фильтрация на $\bar{K}_{0}$ соответствует $p$-градуировке на $\bar{A}$. Рассмотрим стандартные спектральные последовательности $E$ и $\bar{E}$, связанные с этими фильтрованными дифференциально градуированными пространствами (см. [15]). Поскольку $F_{p} K / F_{p-1} K \simeq F_{p} \bar{K} / F_{p-1} \bar{K}$, то

$$
E_{p}^{1}=H\left(F_{p} K / F_{p-1} K\right) \simeq \bar{E}_{p}^{1} .
$$

Для комплекса $\bar{K}$ дифференциал на $\bar{E}^{1}$ всегда нулевой, и, значит, его ацикличность равносильна тому, что $\bar{E}^{1}=0$. Тогда и $E^{1}=0$, и комплекс $K$ также ацикличен.

\section{§5. О рядах Гильберта относительно свободных супералгебр}

В этом параграфе полученные результаты применяются к вопросу о вычислении рядов Гильберта четной и нечетной частей относительно свободной супералгебры. В частности, дана формула, позволяюшая выразить такие ряды Гильберта через ряды Гильберта относительно свободных супералгебр. 
Пусть $W$ - произвольное многообразие. Если $Y=\left\{y_{1}, \ldots, y_{m}\right\}$ - множество четных переменных, а $Z=\left\{z_{1}, \ldots, z_{n}\right\}$ - множество нечетных переменных, то относительно свободную супералгебру $F^{W^{-}}(Y \sqcup Z)$ будем обозначать через $F_{m, n}^{W}$ или просто $F_{m, n}$. Так, $F_{m, 0}=F_{m}$ - относительно свободная алгебра многообразия $W$, а $F_{0, n}-$ внешняя алzебра этого многообразия. Присвоив порождающим степень 1 , можно задать стандартную градуировку на $F_{m, n}$ : степени элемента $x \in F_{m, n}$ в такой градуировке мы будем обозначать через $e(x)$, а градуированные компоненты - верхним индексом в скобках. Все ряды Гильберта ниже вычисляются относительно этой градуировки.

Всякая алгебра $F=F_{m, n}$ разлагается в прямую сумму градуированных подпространств четных и нечетных элементов: $F=F^{0} \oplus F^{1}$. При этом четная часть $F^{0}$ является градуированной подалгеброй $F$ и принадлежит $W$, а нечетная часть $F^{1}$ представляет собой градуированный модуль над четной.

ТЕОРема 8. При $m \geqslant n$ ряды Гильберта градуированных пространств $F^{0}$ и $F^{1}$ связаны соотночением:

$$
F^{0}(t)-F^{1}(t)=F_{m-n, 0}(t)
$$

(здесъ $\left.F_{0,0}(t)=0\right)$.

ДокАЗАТЕЛЬСТво. Пусть $m>n$ (при $m=n$ доказательство аналогично). Положим $A=F^{W}(Y)=F_{m}$ и рассмотрим двусторонний идеал $J \triangleleft A$, порожденный множеством $\rho=\left\{y_{1}, \ldots, y_{n}\right\}$. Поскольку $A / J \simeq F^{W}\left(\left\{y_{n+1}, \ldots, y_{m}\right\}\right) \simeq$ $F_{m-n}$, то $A \simeq \bar{A}$ и $K^{W}(\bar{A}, \rho) \simeq K^{W}(A, \rho) \simeq F_{m, n}=F$. Гомологическая градуировка на этой алгебре задается так: $\left|y_{i}\right|=0,\left|z_{i}\right|=1$, и потому четные и нечетные части относительно гомологической и стандартной градуировок совпадают. Согласно теореме 7 такой комплекс является резольвентой алгебры $A / J \simeq F_{m-n}$ :

$$
0 \longleftarrow F_{m-n} \longleftarrow F_{(0)} \longleftarrow F_{(1)} \longleftarrow \cdots
$$

(нижний индекс в скобках обозначает компоненту в гомологической градуировке). Рассмотрим $k$-ю компоненту в стандартной градуировке:

$$
0 \longleftarrow F_{m-n}^{(k)} \longleftarrow F_{(0)}^{(k)} \longleftarrow F_{(1)}^{(k)} \longleftarrow \cdots .
$$

По формуле Эйлера получаем: $\operatorname{dim} F_{m-n}^{(k)}=\operatorname{dim} F^{0,(k)}-\operatorname{dim} F^{1,(k)}$, откуда немедленно следует требуемое равенство для рядов Гильберта.

СлЕДСТВИЕ 9. При $m \geqslant n$ рядь Гильберта четной и нечетной частей алгебры $F=F_{m, n}^{W}$ вычисляются через ряды Гильберта относительно свободных $W$-супералгебр по формулам:

$$
F^{0}(t)=\frac{F_{m, n}(t)+F_{m-n, 0}(t)}{2}, \quad F^{1}(t)=\frac{F_{m, n}(t)-F_{m-n, 0}(t)}{2} .
$$

В некоторых многообразиях $W$ ряды Гильберта всех относительно свободных супералгебр рациональны: например, как сообшил автору А. Я. Белов, его теорема о рациональности ряда Гильберта любой ассоциативной относительно свободной алгебры [16] обобшается и на случай относительно свободных супералгебр. Таким образом, имеем 
СЛЕДСТВИЕ 10. Пусть многообразие $W$ таково, что ряд Гильберта любой относительно свободной супералгебры рачионален (например, $W \subset \mathrm{Ass}$ ). Тогда при $m \geqslant n$ рядь Гильберта четной и нечетной частей относительно свободной супералгебрь $F_{m, n}^{W}$ рачиональны.

\section{§6. Случай свободных специальных йордановых алгебр}

Напомним (см. [11]), что специальная йорданова алгебра $A^{(+)}$- это ассоциативная алгебра $A$ с умножением $a \circ b=\frac{1}{2}(a b+b a)$. Изучаются также $с п е ц и-$ альные йордановы супералгебры: в этом случае на имеющих четность элементах ассоциативной супералгебры $A$ умножение определяется формулой $a \circ b=$ $\frac{1}{2}\left(a b+(-1)^{\operatorname{deg} a \cdot \operatorname{deg} b} b a\right)$. Если $A$ - свободная ассоциативная (супер)алгебра с множеством порождающих $X$, то свободной специальной йордановой (супер)алгеброй $S J[X]$ называется подалгебра в $A^{(+)}$, порожденная $X$. Ее элементы в $A$ называются йордановыми элементами.

Специальные йордановы алгебры не образуют многообразие (хотя и образуют квазимногообразие), поэтому описанные выше конструкции для них не применимы. Тем не менее, благодаря их тесной связи с ассоциативными алгебрами результаты последнего параграфа удается перенести и на этот случай.

ЛЕмма 11. Пусть на градуированной ассочиативной супералгебре А задано дифферениирование $d$ нечетной степени. Тогда $A^{(+)}$такжсе дифференииально градуирована относительно $d$. Eсли $A=F^{\mathrm{Ass}^{-}}(X)$ u $d X \subset S J[X]$, то $S J[X]$ замкнута относительно такого дифферениирования.

ДокАЗАТЕЛьСтво. В обоих случаях достаточно показать, что дифференцирование $d$ удовлетворяет формуле Лейбница относительно умножения “о”. Действительно, пусть $a$ и $b$ - четности (йордановых) элементов $x$ и $y$. Тогда

$$
\begin{aligned}
d(x \circ y) & =\frac{1}{2}\left(d x \cdot y+(-1)^{a} x \cdot d y+(-1)^{a b} d y \cdot x+(-1)^{b(a+1)} y \cdot d x\right) \\
& =(d x) \circ y+(-1)^{a} x \circ d y
\end{aligned}
$$

Обозначим через $F=F_{m, n}$ свободную ассоциативную, а через $S=S J_{m, n}-$ свободную йорданову супералгебру с $m$ четными и $n$ нечетными порождающими. Как и прежде, для супералгебры $A$ через $A^{0}$ и $A^{1}$ обозначаются пространства четных и нечетных элементов.

ТЕОРема 12. При $m \geqslant n$ ряды Гильберта градуированных пространств $S^{0}$ и $S^{1}$ связаны соотношением:

$$
S^{0}(t)-S^{1}(t)=S J_{m-n, 0}(t)
$$

(здесь, как и выцие, $S J_{0,0}(t)=0$ ). 
ДокаЗАТЕЛЬСтво. Пусть $X=Y \sqcup Z$, где $Y$ и $Z$ - те же, что и в предыдушем параграфе. Если $A=F^{\mathrm{Ass}}(Y)=F_{m, 0}$ и $\rho=\left\{y_{1}, \ldots, y_{n}\right\}$, то комплекс $K^{\mathrm{Ass}}(A, \rho)$ как алгебра изоморфен $F$. Согласно лемме 11 алгебра $S$ является его подкомплексом. При этом факторалгебра $S$ по идеалу, порожденному в $S$ множеством $\rho$, изоморфна $S J_{m-n, 0}$. Следовательно, чтобы применить те же рассуждения, что и в доказательстве теоремы 8 , достаточно обосновать ацикличность комплекса $S$ в положительных степенях.

Действительно, как сказано в доказательстве леммы 6 , дифференциал $d$ на $F=K^{\text {Ass }}(A, \rho)$ в положительных степенях гомотопен тождественному отображению, причем гомотопия в каждой степени пропорциональна дифференцированию $\delta$ нечетной степени такому, что $\delta(\rho)=Z$. По лемме 11 алгебра $S$ выдерживает такое дифференцирование и, следовательно, ограничение $d$ на $S$ также гомотопно (в положительных степенях) тождественному отображению. Таким образом, подкомплекс $S$ ацикличен в положительных степенях, что и требовалось.

СлЕДСТВИЕ 13. При $m \geqslant n$ для рядов Гильберта четной и нечетной частей специальной йордановой супералгебры $S=S J_{m, n}$ справедливь формуль:

$$
\begin{aligned}
& S^{0}(t)=\frac{S J_{m, n}(t)+S J_{m-n, 0}(t)}{2}, \\
& S^{1}(t)=\frac{S J_{m, n}(t)-S J_{m-n, 0}(t)}{2} .
\end{aligned}
$$

\section{Список литературы}

1. Бурбаки Н. Гомологическая алгебра. Новосибирск: Наука, 1987.

2. Jósefiak $T$. Tate resolutions for commutative graded algebras over a local ring // Fund. Math. 1972. V. 74. P. 209-231.

3. Голод Е. С., Шафаревич И. Р. О башне полей классов // Изв. АН СССР. Сер. матем. 1964. T. 28. № 2. C. 261-272.

4. Anick D. Non-commutative graded algebras and their Hilbert series // J. Algebra. 1982. V. 78. P. $120-140$.

5. Anick D. Inert sets and the Lie algebra associated to a group // J. Algebra. 1987. V. 111. P. 154-165.

6. Голод E. С. Некоммутативные полные пересечения и гомологии комплекса Шафаревича // УМH. 1997. Т. 52. № 4. C. 201-202.

7. Halperin S., Lemaire J.-M. Suites inertes dans les algèbres de Lie graduées // Math. Scand. 1987. V. 61. № 1. P. 39-67.

8. Бояркин A.A. Деформации и когомологии в многообразиях алгебр // Дис.... канд. физ.-матем. наук. М., 1977.

9. Кемер А.Р. Многообразия и $\mathbb{Z}_{2}$-градуированные алгебры // Изв. АН СССР. Сер. матем. 1984. Т. 48. № 5. С. 1042-1059.

10. Бояркин А. А. Расширения РІ-алгебр // Матем. заметки. 1986. Т. 40. №6. С. 705-712.

11. Кузъмин E.H., Шестаков И.П. Неассоциативные структуры // Итоги науки и техники. Совр. пробл. матем. Фундам. напр. Т. 57. М.: ВИНИТИ, 1989. С. 179-266.

12. Koszul J. L. Sur un type d'algèbres différentielles en rapport avec la transgression // Colloque de topologie, Brussels, 1950. P. 73-81.

13. Кемер А. Р. Замечание о стандартном тождестве // Матем. заметки. 1978. Т. 23. № 5 . C. $753-757$.

14. Stanley R. P. Hilbert function of graded algebras // Adv. Math. 1978. V. 28. P. 57-83. 
15. Маклейн С. Гомология. М.: Мир, 1966.

16. Белов А.Я. О рациональности рядов Гильберта относительно свободных алгебр // УМН. 1997. Т. 52. № 2. С. 153-154.

Московский государственньй университет им. М.В. Ломоносова
Поступила в редакцию 15.01 .1998 